\title{
Icariin promotes wound healing by enhancing the migration and proliferation of keratinocytes via the AKT and ERK signaling pathway
}

\author{
BOBIN MI, JING LIU, GUOHUI LIU, WU ZHOU, YI LIU, LIANGCONG HU, \\ LIMING XIONG, SHUNAN YE and YONGCHAO WU \\ Department of Orthopedics, Union Hospital, Tongji Medical College, \\ Huazhong University of Science and Technology, Wuhan, Hubei 430022, P.R. China
}

Received January 10, 2018; Accepted May 3, 2018

DOI: $10.3892 / \mathrm{ijmm} .2018 .3676$

\begin{abstract}
Icariin is a traditional Chinese drug that has long been used to treat various diseases. In the present study, the effect of icariin was investigated on cutaneous wound healing. Using in vitro experiments, it was demonstrated that icariin significantly promoted the migration and proliferation of keratinocytes via the activation of AKT serine/threonine kinase 1 (AKT) and extracellular signal-regulated kinase (ERK). Inhibition of AKT or ERK reversed the effects of icariin on the proliferation and migration of keratinocytes. In addition, icariin inhibited the production of interleukin (IL)- 6 and tumor necrosis factor (TNF)- $\alpha$ and induced the production of IL-10. Finally, animal experiments demonstrated that icariin treatment accelerated the wound closure rate. The present findings revealed that icariin may be a promising drug to promote the migration and proliferation of keratinocytes, and to accelerate the healing of skin wounds, through its role in the upregulation of AKT and ERK signaling.
\end{abstract}

\section{Introduction}

Wound healing is a highly complex process, associated with multiple biochemical pathways and a variety of cellular activities, such as re-epithelialization (1) and angiogenesis (2). Following trauma, keratinocytes, the predominant cell type in the epidermis, are activated to contribute to the repair of cutaneous wounds (3). The activated keratinocytes participate in re-epithelialization during wound healing via a series of cellular activities, including cell migration and proliferation (4).

Correspondence to: Dr Guohui Liu or Dr Wu Zhou, Department of Orthopedics, Union Hospital, Tongji Medical College, Huazhong University of Science and Technology, 1277, Jiefang Avenue, Wuhan, Hubei 430022, P.R. China

E-mail: liuguohui@hust.edu.cn

E-mail: 472976834@qq.com

Key words: icariin, keratinocytes, signaling pathway
Therefore, increasing the efficiency of migration and proliferation of keratinocytes may accelerate the wound healing rate. However, keratinocyte migration and proliferation remain not fully elucidated, and therefore reliable and effective drugs that could accelerate the above process have yet to be developed.

Icariin, a well-known traditional Chinese drug, is extracted from Herba Epimedii. The pharmacological and biological effects of icariin, including anti-inflammatory (5), antitumor (6) and neuroprotective (7) effects, have been confirmed by previous studies. Its effects on keratinocytes, however, remain unclear. Previous studies have reported that icariin treatment significantly induces the phosphorylation of AKT serine/threonine kinase 1 (AKT) and extracellular signal-regulated kinase (ERK) in dopaminergic EMS 23.5 cells (8). Deng et al (9) have also reported that icariin significantly upregulates the level of phosphorylated (p)-AKT in rat nucleus pulposus cells. As the phosphorylation of AKT and ERK are closely associated with the migration and proliferation of keratinocytes (10), it is logical to hypothesize that icariin could promote keratinocyte migration and proliferation, thus exerting a beneficial effect on wound healing. Thus, the aim of the present study was to examine the effects of icariin on keratinocytes, and to further investigate the medicinal value of icariin for the treatment of skin wounds.

In the present study, it was demonstrated that icariin significantly accelerated the migration and proliferation of keratinocytes. Furthermore, these processes were demonstrated to be mediated through the activation of ERK and AKT signaling, and to be accompanied by the upregulation of proliferation-associated proteins. In addition, treatment with icariin accelerated wound closure in vivo. These observations indicate that icariin may be a suitable drug for the treatment of skin wounds.

\section{Materials and methods}

Cell culture and reagents. HaCaT cells were obtained from the Type Culture Collection of Chinese Academy of Sciences (Shanghai, China). Cells were grown in Dulbecco's modified Eagle's medium (DMEM; HyClone; GE Healthcare Life Sciences, Logan, UT, USA) containing $10 \%$ heat-inactivated fetal bovine serum (FBS; Gibco; Thermo Fisher Scientific, Inc., 
Waltham, MA, USA $)$ and $1 \%$ antibiotic-antimycotic $(100 \mu \mathrm{g} / \mathrm{ml}$ streptomycin and $100 \mathrm{U} / \mathrm{ml}$ penicillin) and incubated at $37^{\circ} \mathrm{C}$ and $5 \% \mathrm{CO}_{2}$. A Cell Counting Kit-8 (CCK-8) was purchased from MedChemExpress (Monmouth Junction, NJ, USA). Antibodies against GAPDH (cat. no. ab37168), Cyclin D1 (cat. no. ab134175) and Cyclin D3 (cat. no. ab63535) were purchased from Abcam (Cambridge, UK). Antibodies against of AKT (cat. no. 4060), p-Akt (cat. no.\#4691), ERK (cat. no. 4695) and p-ERK (cat. no. 4370) were purchased from Cell Signaling Technology, Inc. (Danvers, MA, USA). The second antibodies against HRP-Goat anti Rabbit (cat. no. AS1107; 1:10,000) and HRP-Goat anti Mouse (cat. no. AS1106; 1:10,000) were purchased from Aspen Biotechnology Co., Ltd. (Wuhan, China). An Akt inhibitor (MK-2206 2HCl) and an ERK inhibitor (GDC-0994) were purchased from Selleck Chemicals (Houston, TX, USA). ELISA kits (IL-6, IL-10, TNF- $\alpha$; cat. nos. HM10205, HM10203 and HM10001 respectively) were purchased from Bio-Swamp Life Science (Shanghai, China). Transwell chambers with $8 \mu \mathrm{m}$ pores were purchased from Corning, Inc. (Corning, NY, USA).

CCK-8 assay. The effect of icariin on $\mathrm{HaCaT}$ cell viability was evaluated by CCK- 8 assay. Briefly, $1 \times 10^{4}$ cells in $100 \mu \mathrm{l}$ were seeded in each well of a 96-well plate and incubated for $24 \mathrm{~h}$ at $37^{\circ} \mathrm{C}$ with $5 \% \mathrm{CO}_{2}$. Various concentrations of icariin as indicated $(0-60 \mu \mathrm{M})$ were dissolved in fresh medium and added to each well. After 24, 48 and $72 \mathrm{~h}$ of incubation, $10 \mu \mathrm{l}$ of CCK-8 reagent was added to each well, and the cells were further incubated for $2 \mathrm{~h}$. The absorbance of each well was measured using a microplate reader at $450 \mathrm{~nm}$. The viability of different groups was evaluated by comparing their optical density (OD) values with that of the control group. The experiment was conducted in triplicate.

Transwell assay. The effect of icariin on $\mathrm{HaCaT}$ cell migration was assessed with a Transwell migration assay. After HaCaT cells were starved in serum-free medium for $24 \mathrm{~h}, 1 \times 10^{5}$ cells in $200 \mu \mathrm{l}$ serum-free growth medium were added to the upper chambers of a 24-well Transwell plate. A total of $500 \mu \mathrm{l}$ of medium containing $10 \%$ FBS was added to each lower chamber, as a chemoattractant. PBS (vehicle control) or $30 \mu \mathrm{M}$ icariin were added into the lower chamber. Following incubation for $24 \mathrm{~h}$ at $37^{\circ} \mathrm{C}$, the migrated $\mathrm{HaCaT}$ cells were fixed with methanol, and stained with $0.1 \%$ crystal violet. Images were obtained using a light microscope (Olympus Corporation, Tokyo, Japan) at x10 magnification. Cells were counted in five different optical fields using Image-Pro Plus 6.0 software (Media Cybernetics, Inc., Rockville, MD, USA). The experiment was conducted in triplicate.

Western blot assay. For evaluation of the ERK and AKT pathways, cells were treated with PBS or $30 \mu \mathrm{M}$ icariin for $20 \mathrm{~min}$. For evaluation of the cyclin proteins, cells were treated with PBS, $30 \mu \mathrm{M}$ icariin, $10 \mu \mathrm{M}$ MK-2206 $2 \mathrm{HCl}+30 \mu \mathrm{M}$ icariin, or $10 \mu \mathrm{M}$ GDC-0994 $+30 \mu \mathrm{M}$ icariin for $24 \mathrm{~h}$. Following treatments as indicated, total protein was extracted with RIPA lysis buffer (Wuhan Boster Biological Technology, Ltd., Wuhan, China) and protease inhibitor cocktail (Roche Diagnostics, Basel, Switzerland) on ice. The protein concentration was measured using a BCA protein assay kit (Thermo Fisher
Scientific, Inc., Waltham, MA, USA). A total of $40 \mu \mathrm{g}$ of protein from each sample was separated with 10\% SDS-PAGE, and electrophoretically transferred to a polyvinylidene fluoride membrane. Following transfer, the membrane was blocked with 5\% fat-free milk in PBST (PBS containing 0.05\% Tween-20; Wuhan Boster Biological Technology, Ltd.) for $1 \mathrm{~h}$ at room temperature and was incubated with the primary antibodies targeting AKT $(1: 1,000)$, p-AKT $(1: 3,000)$, ERK $(1: 3,000)$, p-ERK (1:1,000), Cyclin D1 (1:3,000), Cyclin D3 $(1: 1,000)$ and GAPDH $(1: 10,000)$ at $4^{\circ} \mathrm{C}$ overnight. Subsequently, the membrane was incubated with an HRP-conjugated secondary antibody for $1 \mathrm{~h}$ at room temperature. Protein bands were visualized using an Odyssey Infrared Imaging System (LI-COR Biosciences, Lincoln, NE, USA). ImageJ software (National Institutes of Health, Bethesda, MD, USA) was used for quantitative analysis of scanned densitometric values of the proteins as ratios to GAPDH (used as a loading control).

Reverse transcription-quantitative polymerase chain reaction (RT-qPCR). Cells were treated with PBS, $30 \mu \mathrm{M}$ icariin, $10 \mu \mathrm{M}$ MK-2206 $2 \mathrm{HCl}+30 \mu \mathrm{M}$ icariin, or $10 \mu \mathrm{M}$ GDC-0994 $+30 \mu \mathrm{M}$ icariin for $6 \mathrm{~h}$. Following treatments, total RNA was extracted from each group using RNA Iso Plus reagent (Invitrogen; Thermo Fisher Scientific, Inc.), according to the manufacturer's instructions. RNA was reverse-transcribed into cDNA using the PrimeScript RT Reagent kit (Takara Bio., Inc., Otsu, Japan). qPCR was performed with SYBR Master Mix (Takara Bio., Inc.) using a StepOne Real-Time PCR System (Life Technologies; Thermo Fisher Scientific, Inc.). The thermocycling conditions were as follows: Pre-denaturing at $95^{\circ} \mathrm{C}$ for $1 \mathrm{~min}$, amplification for 40 cycles by denaturing at $95^{\circ} \mathrm{C}$ for $15 \mathrm{sec}$, annealing at $58^{\circ} \mathrm{C}$ for $20 \mathrm{sec}$ and extension at $72^{\circ} \mathrm{C}$ for $45 \mathrm{sec}$, followed by a final dissociation cycle of $95^{\circ} \mathrm{C}$ for $15 \mathrm{sec}, 60^{\circ} \mathrm{C}$ for $1 \mathrm{~min}$ and $95^{\circ} \mathrm{C}$ for $15 \mathrm{sec}$. The relative mRNA quantity of each gene was normalized to GAPDH and calculated using the $2^{-\Delta \Delta C q}$ method (11). The primer sequences were: Tumor necrosis factor (TNF)- $\alpha$, forward, 5'-CTCTTCTCCTTCCTGATCGTGG-3' and reverse, 5'-CTTGTCACTCGGGGTTCGAG-3'; interleukin (IL)-6, forward, 5'-TCAGCCCTGAGAAAGGAGACAT-3' and reverse, 5'-GCTCTGGCTTGTTCCTCACTACT-3'; IL-10, forward, 5'-AACCTGCCTAACATGCTTCG-3' and reverse, 5'-GAG TTCACATGCGCCTTGAT-3'; and GAPDH, forward, 5'-CAT CATCCCTGCCTCTACTGG-3' and reverse, 5'-GTGGGTGTC GCTGTTGAAGTC-3'. The experiment was conducted in three independent biological replicates.

ELISA. The levels of IL-6, IL-10 and TNF- $\alpha$ secreted by HaCaT cells were measured using ELISA kits. The $\mathrm{HaCaT}$ cells were seeded into a 24-well plate at a density of $2 \times 10^{4}$ cells per well, incubated overnight, and then treated with PBS, $30 \mu \mathrm{M}$ icariin, $10 \mu \mathrm{M}$ MK-2206 $2 \mathrm{HCl}+30 \mu \mathrm{M}$ icariin, or $10 \mu \mathrm{M}$ GDC-0994 + $30 \mu \mathrm{M}$ icariin. Following treatment for $24 \mathrm{~h}$, supernatants were collected and the release of IL-6, IL-10 and TNF- $\alpha$ into the media was quantified using the ELISA kits, according to the manufacturer's instructions. The experiment was conducted in triplicate.

Wound healing animal model. A total of ten male Sprague Dawley rats (age, 4 weeks) were purchased from the Center of Experimental Animals, Tongji Medical College, Huazhong 
University of Science and Technology (Wuhan, China). The rats were housed under standard laboratory conditions with a 12-h light/dark cycle, with food and water supplied. All animal studies were conducted under the review and with the approval of the Ethics Committee of Tongji Medical College (Wuhan, China). All experiments were performed in accordance with the relevant guidelines and regulations of Tongji Medical College.

The rats were randomly divided into two groups ( $\mathrm{n}=5$ per group): The control group and the icariin group. Rats were anesthetized using $10 \%$ chloral hydrate $(0.3 \mathrm{ml} / 100 \mathrm{~g}$ body weight). The fur on the back of each rat was clipped, and a $1 \times 1 \mathrm{~cm}$ full-thickness wound was generated on each rat. A total of $1 \mathrm{ml}$ containing either $30 \mu \mathrm{M}$ icariin (icariin group) or PBS (control group) was injected into the surrounding tissue of the wound each day for a total of 10 days. The wound area was monitored by capturing digital photographs at 0 , 4, 7 and 10 days post-injury and quantified using Image-Pro Plus 6.0 software (Media Cybernetics). To ensure that the wound areas could be compared between the two groups, digital photos were captured using a ruler for reference. Three authors collected the wound area data independently and the mean value was used for statistical analysis. The rate of wound closure was calculated using the following formula: Wound closure $(\%)=[($ original wound area-open area on at time-point)/original wound area] $\times 100 \%$.

Hematoxylin and eosin $(H \& E)$ staining. Full thickness skin samples of all wounds were collected for histological analysis after 10 days of PBS or icariin was applied on the wound of animals. Tissue samples were fixed in $10 \%$ neutral-buffered formalin solution for $24 \mathrm{~h}$, then transferred and stored in $70 \%$ ethyl alcohol until paraffin embedded. Paraffin-embedded samples were cut into $4 \mu \mathrm{m}$ thick sections for hematoxylin and eosin staining, under standard protocols.

Epidermal thickness assessment. The epidermal thickness was measured using the H\&E sections. Measurements and quantification were performed with Image-Pro Plus 6.0 software (Media Cybernetics) under $\mathrm{x} 4$ magnification. Epidermal thickness was evaluated in three random fields of each sample. Three independent observers completed these analyses. The mean value of each measurement was used for statistical analysis.

Statistical analysis. Statistical analysis was performed with SPSS 12.0 software (SPSS Inc., Chicago, IL, USA) and GraphPad Prism 6.0 software (GraphPad Software, Inc., La Jolla, CA, USA). Data are presented as the mean \pm standard deviation. Differences between samples were compared by t-test between two groups or one-way analysis of variance (ANOVA) among multiple groups. The Student-Newman-Keuls post hoc method was used to calculate the $\mathrm{P}$-value for pairwise comparisons following ANOVA. $\mathrm{P}<0.05$ was considered to indicate a statistically significant difference.

\section{Results}

Cytotoxicity of icariin in keratinocytes. To evaluate the effect of icariin on keratinocyte viability, a CCK-8 assay was used to test cell viability. At $24 \mathrm{~h}$ following treatment with icariin, the cell viability in the 5-50 $\mu \mathrm{M}$ icariin treatment groups was higher compared with the group treated with $0 \mu \mathrm{M}$ icariin (Fig. 1). At $48 \mathrm{~h}, 10-40 \mu \mathrm{M}$ icariin significantly increased cell viability, whereas a concentration of $50 \mu \mathrm{M}$ or $60 \mu \mathrm{M}$ icariin did not affect viability (Fig. 1). However, following $72 \mathrm{~h}$ of treatment, $30 \mu \mathrm{M}$ icariin significantly increased cell viability, while $60 \mu \mathrm{M}$ icariin significantly inhibited cell viability, and 40 and $50 \mu \mathrm{M}$ had no effect on cellular viability (Fig. 1). Therefore, the dose of $30 \mu \mathrm{M}$ icariin was selected as optimal in the subsequent experiments in order to prevent cytotoxicity.

Icariin promotes the proliferation of keratinocytes. A CCK-8 assay demonstrated that the proliferation of $\mathrm{HaCaT}$ cells in the icariin group was higher compared with the control group (Fig. 2A). To further confirm the effect of icariin on cell proliferation, the levels of Cyclin D1 and D3, two proteins that are closely associated with cell proliferation, were quantified by western blotting. The results demonstrated that the expression levels of Cyclin D1 and D3 were markedly increased in the icariin treatment group compared with the control group (Fig. 2B).

Icariin promotes the migration of keratinocytes. To investigate the migration ability of the keratinocytes treated with icariin, a Transwell migration assay was performed. Representative images of the Transwell assay results at $24 \mathrm{~h}$ are presented in Fig. 3A. Quantitative analysis of the numbers of migrated cells revealed that the icariin-treated group exhibited a significant increase in migration ability compared with the control group $(\mathrm{P}<0.001$; Fig. 3B).

ERK and AKT are involved in the icariin-mediated promotion of keratinocyte proliferation and migration. To investigate which signaling pathways were involved in the promotion of migration and proliferation by icariin, the activation of the ERK and AKT signaling pathways was assayed by western blotting. The results demonstrated that the levels of p-ERK and p-AKT were significantly increased at $30 \mathrm{~min}$ following treatment with icariin compared with the control group (Fig. 4).

Subsequently, the effect of ERK and AKT inhibitors was assessed on $\mathrm{HaCaT}$ cell migration and proliferation. $\mathrm{HaCaT}$ cells were incubated with the MK-2206 2HCl AKT inhibitor $(10 \mu \mathrm{M})$ or the GDC-0994 ERK inhibitor $(50 \mu \mathrm{M})$ for $2 \mathrm{~h}$ prior to treatment with icariin. The icariin-mediated increase on cell proliferation (Fig. 2) and migration (Fig. 3) was significantly blocked when cells were pretreated with the ERK or the AKT inhibitor. These results indicated that phosphorylation and activation of AKT and ERK were required for the effects of icariin on keratinocytes.

Icariin treatment alters the production of cytokines in keratinocytes. Following treatment of $\mathrm{HaCaT}$ cells with icariin $(30 \mu \mathrm{M})$, the mRNA expression levels of cytokines were detected by RT-qPCR (at $6 \mathrm{~h}$ ), while the levels of secreted cytokines in the cell supernatants were determined by ELISA kits (at $24 \mathrm{~h}$ ). As illustrated in Fig. 5, the IL-6 and TNF- $\alpha$ mRNA expression decreased and the IL-10 mRNA expression increased in the icariin group compared with the control group. At the protein level, icariin inhibited the secretion of IL- 6 and TNF- $\alpha$, and 

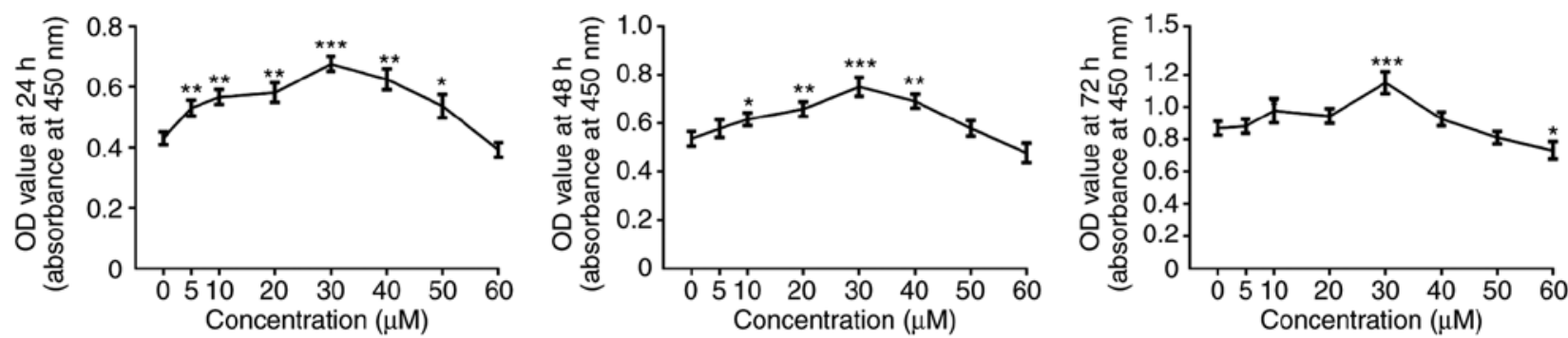

Figure 1. Cytotoxicity of icariin on HaCaT cells. The viability of HaCaT cells treated with 0-60 $\mu \mathrm{M}$ icariin for 24-72 h was analyzed with the CCK-8 assay. Data represent mean \pm standard deviation of three independent experiments. Icariin concentration of $30 \mu \mathrm{M}$ was regarded safe and used for the subsequent experiments. ${ }^{*} \mathrm{P}<0.05,{ }^{* *} \mathrm{P}<0.01$ and ${ }^{* * *} \mathrm{P}<0.001$ compared with untreated control. CCK-8, cell counting kit-8; OD, optical density.
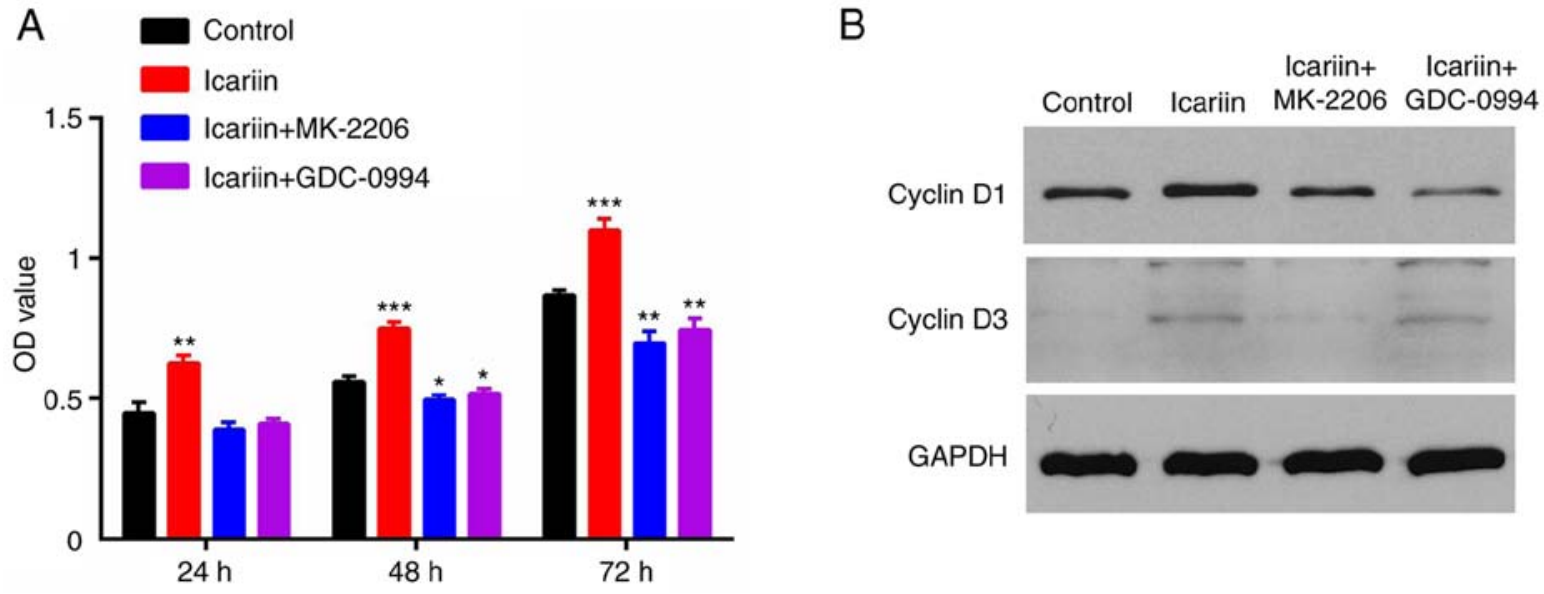

Figure 2. Icariin promotes $\mathrm{HaCaT}$ cell proliferation. (A) HaCaT cells were pretreated with MK-2206, GDC-0994, or medium alone without inhibitors for $2 \mathrm{~h}$ Cells were then incubated with $30 \mu \mathrm{M}$ icariin for 24,48 and $72 \mathrm{~h}$, and cell viability was measured by CCK-8 assay. (B) Western blot analysis of Cyclin D1 and D3 protein expression levels in HaCaT cells following treatment with medium alone (control), icariin, icariin with MK-2206 or icariin with GDC-0994. Data represent mean \pm standard deviation of three independent experiments. ${ }^{*} \mathrm{P}<0.05,{ }^{* *} \mathrm{P}<0.01$ and ${ }^{* * *} \mathrm{P}<0.001$ vs. untreated control. CCK-8, cell counting kit-8; OD, optical density.

A
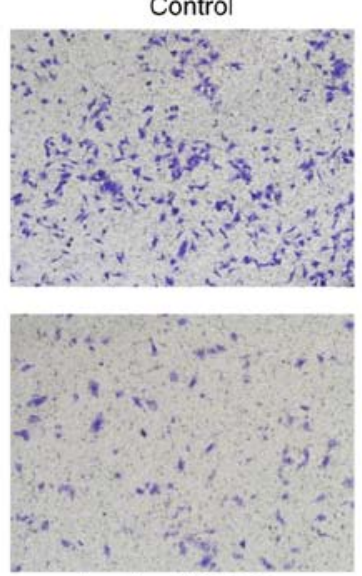

Icariin+MK-2206
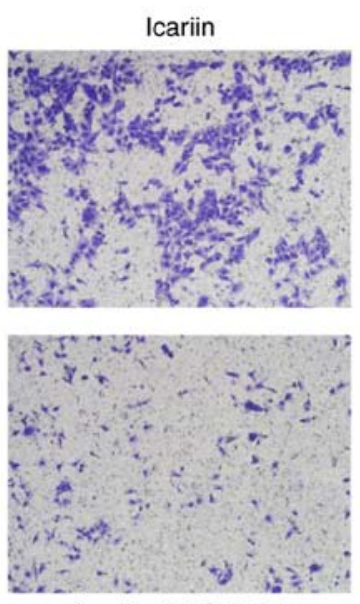

Icariin+GDC-0994

\section{B}

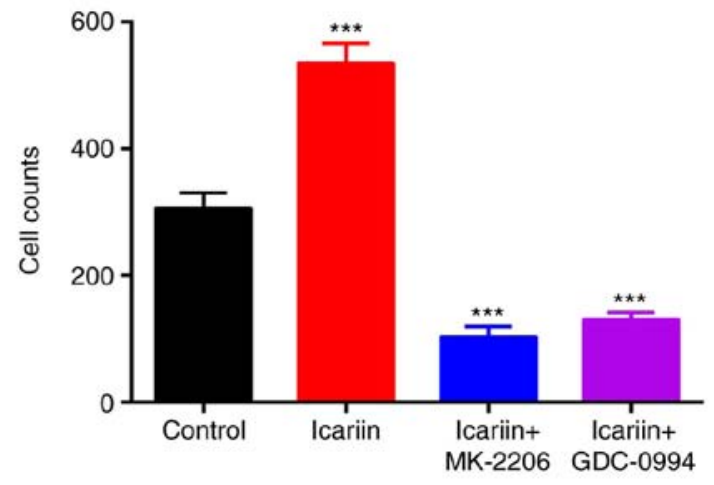

Figure 3. Icariin promotes HaCaT cell migration. Cell migration was measured by Transwell assay, where medium alone, icariin, icariin with MK-2206 or icariin with GDC-0994 were added to the lower chamber and 5x10 4 cells/well were added into the upper chamber. Following incubation for $24 \mathrm{~h}$, the migrated cells on the membrane were stained and counted under a microscope. (A) Representative images of the migrated cells in each treatment group. (B) Quantification of the numbers of migrated cells in each group. Data represent mean \pm standard deviation of three independent experiments. ${ }^{* * *} \mathrm{P}<0.01$ vs. untreated control.

induced the secretion of IL-10 (Fig. 6). Furthermore, we investigated if the AKT and ERK signaling pathway was involved in this process. Cells were pretreated for $2 \mathrm{~h}$ with $10 \mu \mathrm{M}$ MK-2206 $2 \mathrm{HCl}$ or $50 \mu \mathrm{M}$ GDC-0994 prior to treatment with
$30 \mu \mathrm{M}$ icariin. AKT and ERK inhibition resulted in a significant decrease of the stimulatory effects of icariin to elicit the production of IL-10, while the production of IL- 6 and TNF- $\alpha$ were increased compared with icariin treatment alone (Figs. 5 and 6). 

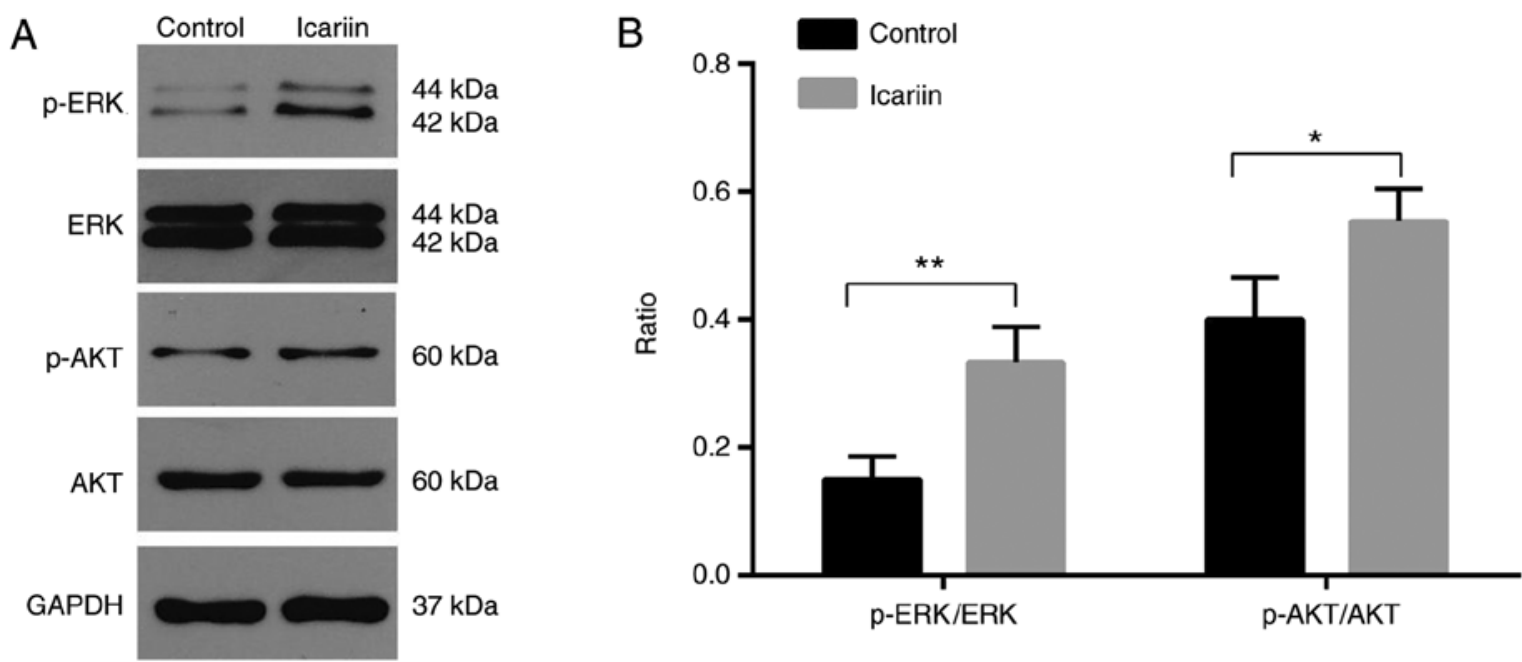

Figure 4. Icariin induces phosphorylation of ERK and AKT. HaCaT cells were stimulated with $30 \mu \mathrm{M}$ of icariin for 30 min, and then the protein expression levels of p-ERK, total ERK, p-AKT and total AKT were determined by western blot analysis. GAPDH was used as loading control. (A) Representative blots. (B) Quantification of the ratio of the intensity of phosphorylated protein divided by the total protein. Data represent mean \pm standard deviation of three independent experiments. ${ }^{*} \mathrm{P}<0.05,{ }^{* *} \mathrm{P}<0.01$ compared with untreated control. ERK, extracellular signal-regulated kinase; AKT, AKT serine/threonine kinase 1; p-, phosphorylated.

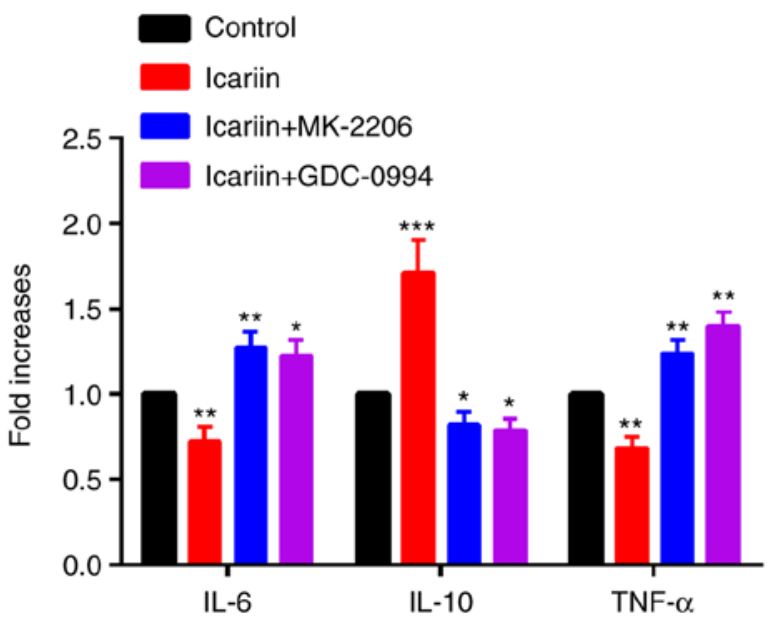

Figure 5. Effects of icariin on the gene expression of inflammatory cytokines in $\mathrm{HaCaT}$ cells. HaCaT cells were pretreated with MK-2206, GDC-0994, or medium alone without inhibitors for $2 \mathrm{~h}$. Cells were then incubated with icariin for $6 \mathrm{~h}$. The mRNA expression levels of IL-6, IL-10 and TNF- $\alpha$ were measured by reverse transcription-quantitative polymerase chain reaction. Data represent mean \pm standard deviation of three independent experiments. ${ }^{*} \mathrm{P}<0.05,{ }^{* *} \mathrm{P}<0.01$ and ${ }^{* * *} \mathrm{P}<0.001$ vs. untreated control. IL, interleukin TNF, tumor necrosis factor.

Icariin promotes wound healing in vivo. To investigate whether icariin was beneficial for wound closure in vivo, experimental rats were divided into two groups, receiving either vehicle or icariin treatment following the induction of skin wounds. The icariin group displayed a significantly accelerated wound closure rate compared with the control group (Fig. 7). Furthermore, histopathological analysis and microscopic assessment of epidermal thickness was performed on the final wound closure sites. The epidermis was significantly thicker in the icariin group compared with the control group (Fig. 8). These findings demonstrated that icariin was beneficial to wound repair via stimulation of re-epithelialization and keratinocyte proliferation.

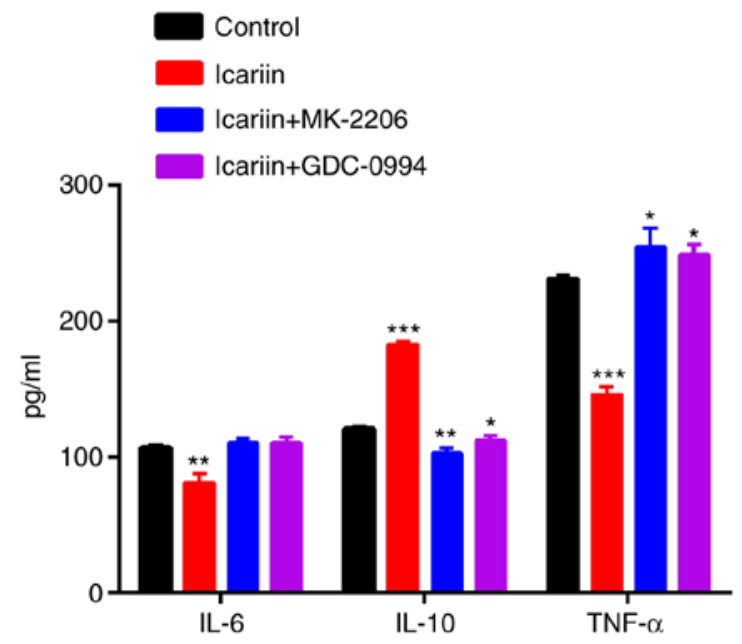

Figure 6. Effects of icariin on inflammatory cytokine secretion in $\mathrm{HaCaT}$ cells. HaCaT cells were pretreated with MK-2206, GDC-0994, or medium alone without inhibitors for $2 \mathrm{~h}$. Cells were then incubated for $24 \mathrm{~h}$ with icariin. The concentrations of released IL-6, IL-10 and TNF- $\alpha$ in the culture supernatants were measured by ELISA. Data represent mean \pm standard deviation of three independent experiments. ${ }^{*} \mathrm{P}<0.05,{ }^{* *} \mathrm{P}<0.01$ and ${ }^{* * * *} \mathrm{P}<0.001$ vs. untreated control. IL, interleukin; TNF, tumor necrosis factor.

\section{Discussion}

The cytotoxic effects of icariin on several cell types, including SW 1353 chondrosarcoma (12) and human nucleus pulposus (5) cells, have been extensively explored. In those studies, the dose of icariin was varied from 5 to $50 \mu \mathrm{M}$. In the present study, exposure to $60 \mu \mathrm{M}$ icariin for $72 \mathrm{~h}$ reduced cell proliferation, whereas concentrations of $50 \mu \mathrm{M}$ or less had no growth inhibitory effect on $\mathrm{HaCaT}$ cells. Based on the CCK-8 assay in the present study, $30 \mu \mathrm{M}$ icariin was identified as the optimal concentration for promoting cell proliferation at $72 \mathrm{~h}$.

Once a skin wound occurs, keratinocytes migrate to the wound site. The stimulatory role of icariin on keratinocyte 
A
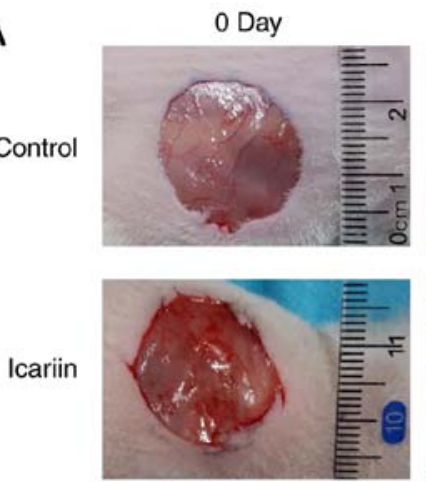
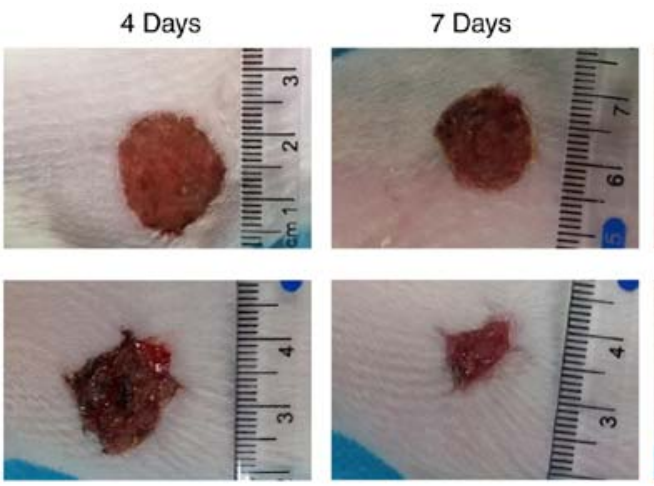

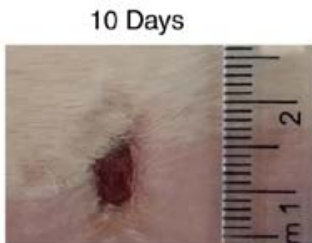

B

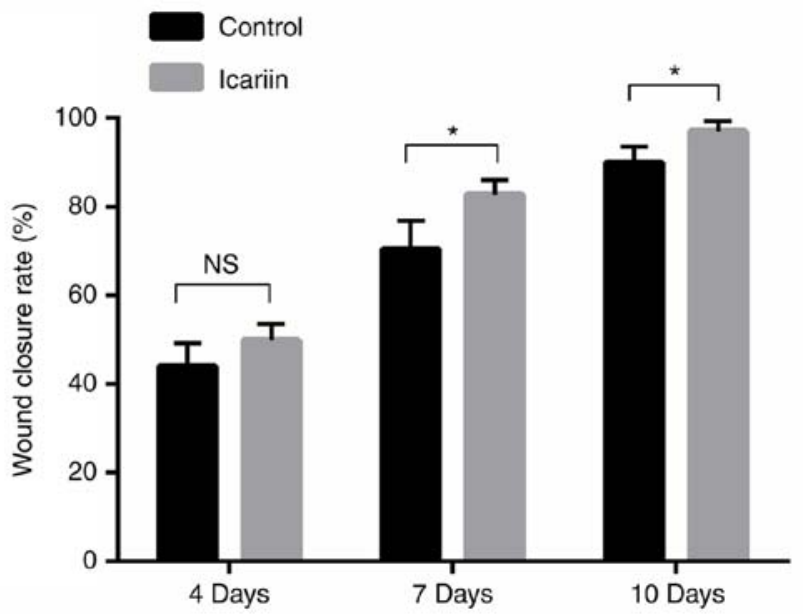

Figure 7. Effect of icariin on wound closure in vivo. The effect of icariin on closure of full-thickness excisional wounds was evaluated in rats. Each wound was treated with PBS or icariin daily for a total of 10 days. The animals were lightly anesthetized and the wound area was photographed and evaluated on the indicated days following injury. (A) Representative images of the wound site in the two treatment groups. (B) Quantification of the wound closure rate as \% of wound area at different time point in comparison to the original wound area. ${ }^{*} \mathrm{P}<0.05$ with comparisons indicated by lines.
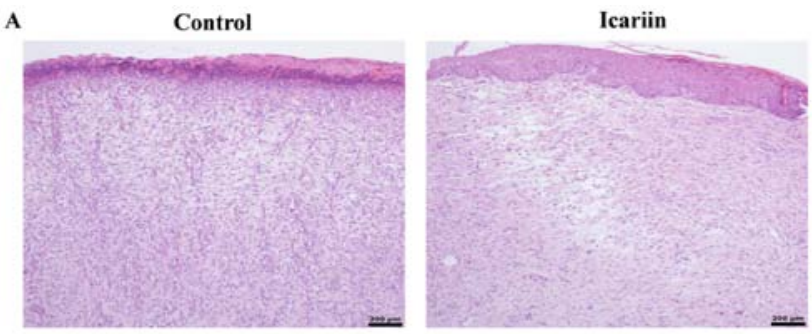

B

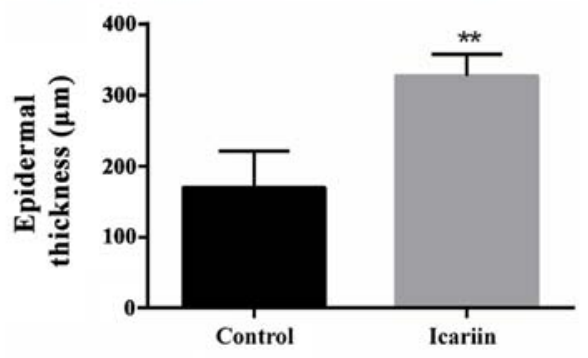

Figure 8. Effects of icariin treatment on epidermal thickness in vivo. (A) Representative images and (B) quantification of epidermal thickness from hematoxylin and eosin stained sections of the wound sites of the animals treated with PBS or icariin. ${ }^{* *} \mathrm{P}<0.01$.

migration was demonstrated in the present study by a Transwell assay. The present results suggested that icariin promoted the migration of keratinocytes. Consistent with the current results, migration is also promoted by icariin in other cell types; icariin could induce human umbilical cord mesenchymal stem cell migration in chronic liver injury in mice (13). The treatment of endothelial progenitor cells with icariin also promoted cell migration (14). The effect of icariin on keratinocyte proliferation was demonstrated in the present study by CCK-8 and western blot assays. In the icariin group, the rate of keratinocyte proliferation was higher compared with the untreated control group. The expression levels of Cyclin D1 and D3, proteins with important roles in cell proliferation, were also upregulated in the icariin group. Additionally, the present in vivo study demonstrated that icariin could accelerate re-epithelialization and wound healing rate. There are various types of cells involved in the process of wound healing. Icariin may also have effects on other cell types, such as fibroblasts and mesenchymal stem cells. The effects of icariin on proliferation of other cell types may also contribute to the accelerated wound healing rate, and this will be further explored in future studies.

In the present study, the mechanisms involved in the effects of icariin on the migration and proliferation of keratinocytes were investigated. Icariin has been reported to exert effects on migration and proliferation in various types of cells through multiple signaling pathways $(15,16)$. AKT and ERK have important roles in the regulation of cell growth, proliferation, differentiation, death and other processes (17-19). Notably, previous studies have reported that AKT and ERK are involved in the migration and proliferation of keratinocytes $(20,21)$, 
indicating that they may be associated with the migration and proliferation of keratinocytes induced by icariin. The present study identified that icariin promoted the phosphorylation of ERK and AKT in keratinocytes. This result was consistent with previous studies regarding the effect of icariin on the phosphorylation of ERK and AKT in other cells types. Furthermore, the present results demonstrated that pretreatment with an ERK or AKT inhibitor significantly inhibited the icariin-induced migration and proliferation of keratinocytes, suggesting that ERK and AKT activation were critical for the effects of icariin. In addition, Cyclin D1 and D3 were significantly downregulated following pretreatment with the AKT or ERK inhibitor.

A number of cytokines and chemokines act as mediators of cell migration, proliferation and differentiation during wound healing. A variety of cytokines are secreted by activated keratinocytes during the proliferation and re-epithelialization phase of wound healing, and act as chemoattractants to activate neighboring keratinocytes (22). IL-10 is a regulatory cytokine produced by various cells types that can inhibit the production of proinflammatory cytokines, such as IL-6 and TNF- $\alpha$ (23). IL-6 derived from macrophages, fibroblasts and keratinocytes, affects granulation tissue formation, re-epithelialization and angiogenesis (24). TNF- $\alpha$ is produced by a variety of cell types, including keratinocytes, macrophages and mast cells. It serves dual roles in decreasing granulation tissue production and collagen fiber arrangement $(25,26)$. However, excessive production of proinflammatory cytokines, such as IL-1 $\beta$, TNF- $\alpha$ and IL-6, may result in harmful inflammation and the risk of sepsis (27). As illustrated in Fig. 5, the mRNA expression of IL- 6 and TNF- $\alpha$ was significantly decreased in the icariin-treated group. Conversely, the mRNA expression of IL-10 was increased in the icariin-treated group. In accordance with this result, IL-6 and TNF- $\alpha$ secreted levels were significantly inhibited in the culture supernatants of icariin-treated $\mathrm{HaCaT}$ cells at $24 \mathrm{~h}$, while the icariin-treated group secreted more IL-10, compared with the control group. These results indicated that icariin, not only inhibited the secretion of proinflammatory cytokines, but also induced keratinocytes to secrete anti-inflammatory cytokines. AKT and ERK have been reported to regulate cytokine production and release, and have been demonstrated to serve an important role in wound repair $(28,29)$. Specific inhibitors for AKT and ERK blocked the downregulation of IL- 6 and TNF- $\alpha$ and the upregulation of IL-10 induced by icariin treatment, suggesting that these kinases were involved in the process of icariin-induced cytokine expression alterations.

Collectively, the present study demonstrated that $30 \mu \mathrm{M}$ icariin was the optimal concentration for promoting growth in $\mathrm{HaCaT}$ cells. Icariin treatment enhanced the migration and proliferation of keratinocytes by activating the AKT and ERK signaling pathways. In addition, icariin altered the expression of cytokines through the AKT and ERK signaling pathways. Furthermore, icariin accelerated wound healing in vivo. Therefore, icariin may be considered a promising drug for the treatment of skin wounds. Further studies are still required to confirm the effect of icariin and its constituent in vivo models.

\section{Acknowledgements}

Not applicable.

\section{Funding}

This study was supported by the National Science Foundation of China (grant no. 81772345), the Science and Technology Department of Hubei Province (grant no. 2016CFB424), and the DevelopmentCenterforMedicalScienceandTechnology National Health andFamily Planning Commission of the People's Republic of China (grant no. ZX-01-C2016024).

\section{Availability of data and materials}

The analyzed datasets generated during the study are available from the corresponding author on reasonable request.

\section{Authors' contributions}

BM and GL designed the study and prepare the manuscript. YL, JL and LH performed the cell experiments. LH and WZ performed the animal experiments. SY and YW analyzed the data. GL revised the manuscript. All authors read and approved the final manuscript.

\section{Ethics approval and consent to participate}

All animal studies were conducted under the review and with the approval of the Ethics Committee of Tongji Medical College (Wuhan, China).

\section{Consent for publication}

Not applicable.

\section{Competing interests}

The authors declare that they have no competing interests.

\section{References}

1. Gugerell A, Kober J, Schmid M, Buchberger E, Kamolz LP and Keck M: Botulinum toxin A: Dose-dependent effect on reepithelialization and angiogenesis. Plast Reconstr Surg Glob Open 4: e837, 2016.

2. Chen X, Zhang M, Wang X, Chen Y, Yan Y, Zhang L and Zhang L: Peptide-modified chitosan hydrogels promote skin wound healing by enhancing wound angiogenesis and inhibiting inflammation. Am J Transl Res 9: 2352-2362, 2017.

3. Kim MH, Wu WH, Choi JH, Kim JH, Jun JH, Ko Y and Lee JH: Galectin-1 from conditioned medium of three-dimensional culture of adipose-derived stem cells accelerates migration and proliferation of human keratinocyte and fibroblasts. Wound Repair Regen: Aug 30, 2017 (Epub ahead of print).

4. Gonzalez AC, Costa TF, Andrade ZA and Medrado AR: Wound healing-A literature review. An Bras Dermatol 91: 614-620, 2016.

5. Hua W, Zhang Y, Wu X, Kang L, Tu J, Zhao K, Li S, Wang K, Song Y, Luo R, et al: Icariin Attenuates Interleukin-1 $\beta$-induced inflammatory response in human nucleus pulposus cells. Curr Pharm Des 23: 6071-6078, 2018.

6. Li X, Sun J, Hu S and Liu J: Icariin induced B16 melanoma tumor cells apoptosis, suppressed tumor growth and metastasis. Iran J Public Health 43: 847-848, 2014.

7. Jiang $\mathrm{MC}$, Chen $\mathrm{XH}$, Zhao $\mathrm{X}$, Zhang $\mathrm{XJ}$ and Chen WF: Involvement of IGF-1 receptor signaling pathway in the neuroprotective effects of Icaritin against MPP(+)-induced toxicity in MES23.5 cells. Eur J Pharmacol 786: 53-59, 2016. 
8. Chen WF, Wu L, Du ZR, Chen L, Xu AL, Chen XH, Teng JJ and Wong MS: Neuroprotective properties of icariin in MPTP-induced mouse model of Parkinson's disease: Involvement of PI3K/Akt and MEK/ERK signaling pathways. Phytomedicine 25: 93-99, 2017.

9. Deng $X$, Chen $S$, Zheng D, Shao Z, Liang $\mathrm{H}$ and $\mathrm{Hu} \mathrm{H}$ : Icariin prevents $\mathrm{H}_{2} \mathrm{O}_{2}$-induced apoptosis via the PI3K/Akt pathway in rat nucleus pulposus intervertebral disc cells. Evid Based Complement Alternat Med 2017: 2694261, 2017.

10. Yoon SW, Lee KP, Kim DY, Hwang DI, Won KJ, Lee DW and Lee HM: Effect of absolute from Hibiscus syriacus L. Flower on wound healing in keratinocyte. Pharmacogn Mag 13: 85-89, 2017.

11. Livak KJ and Schmittgen TD: Analysis of relative gene expression data using real-time quantitative PCR and 2(-Delta Delta C(T)) method. Methods 25: 402-408, 2001.

12. Zeng L, Wang W, Rong XF, Zhong Y, Jia P, Zhou GQ and Li RH: Chondroprotective effects and multi-target mechanisms of Icariin in IL-1 beta-induced human SW 1353 chondrosarcoma cells and a rat osteoarthritis model. Int Immunopharmacol 18: 175-181, 2014.

13. Cui H, Liu Z, Wang L, Bian Y, Li W, Zhou H, Chu X and Zhao Q Icariin-treated human umbilical cord mesenchymal stem cells decrease chronic liver injury in mice. Cytotechnology 69: 19-29, 2017.

14. Tang Y, Jacobi A, Vater C, Zou L, Zou X and Stiehler M: Icariin promotes angiogenic differentiation and prevents oxidative stress-induced autophagy in endothelial progenitor cells. Stem Cells 33: 1863-1877, 2015.

15. Gu ZF, Zhang ZT, Wang JY and Xu BB: Icariin exerts inhibitory effects on the growth and metastasis of KYSE70 human esophageal carcinoma cells via PI3K/AKT and STAT3 pathways. Environ Toxicol Pharmacol 54: 7-13, 2017.

16. Liu Y,Huang L,HaoB,LiH,Zhu S, Wang Q,LiR,Xu Y and Zhang X Use of an osteoblast overload damage model to probe the effect of icariin on the proliferation, differentiation and mineralization of MC3T3-E1 cells through the Wnt/ $\beta$-Catenin signalling pathway. Cell Physiol Biochem 41: 1605-1615, 2017.

17. Sun P, Sun X, Zhao W, Ren M, Zhang C, Wang Z and Xu W: Lemur tyrosine kinase- 3 suppresses growth of prostate cancer via the AKT and MAPK signaling pathways. Cell Physiol Biochem 42: 2582-2592, 2017.

18. Jeong YJ, Hoe HS, Cho HJ, Park KK, Kim DD, Kim CH, Magae J, Kang DW, Lee SR and Chang YC: Suppression of c-Myc enhances $\mathrm{p} 21^{\mathrm{WAF} 1 / \mathrm{CIP1}}$-mediated G1 cell cycle arrest through the modulation of ERK phosphorylation by ascochlorin. J Cell Biochem 119: 2036-2047, 2018.

19. Krajarng A, Chulasiri M and Watanapokasin R: Etlingera elatior extract promotes cell death in B16 melanoma cells via down-regulation of ERK and Akt signaling pathways. BMC Complement Altern Med 17: 415, 2017.
20. Zhou T, Yang Z, Chen Y,Chen Y,Huang Z, You B,Peng Y and Chen J: Estrogen accelerates cutaneous wound healing by promoting proliferation of epidermal keratinocytes via Erk/Akt signaling pathway. Cell Physiol Biochem 38: 959-968, 2016.

21. Zhao B, Liu JQ, Zheng Z, Zhang J, Wang SY, Han SC, Zhou Q, Guan H, Li C, Su LL and Hu DH: Human amniotic epithelial stem cells promote wound healing by facilitating migration and proliferation of keratinocyte via ERK, JNK and AKT signaling pathways. Cell Tissue Res 365: 85-99, 2016.

22. Freedberg IM, Tomic-Canic M, Komine M and Blumenberg M Keratins and the keratinocyte activation cycle. J Invest Dermatol 116: 633-640, 2001.

23. Couper KN, Blount DG and Riley EM: IL-10: The master regulator of immunity to infection. J Immunol 180: 5771-5777, 2008.

24. Feng Y, Sanders AJ, Morgan LD, Harding KG and Jiang WG: Potential roles of suppressor of cytokine signaling in wound healing. Regen Med 11: 193-209, 2016.

25. Gragnani A, Müller BR, Silva ID, Noronha SM and Ferreira LM: Keratinocyte growth factor, tumor necrosis factor-alpha and interleukin-1 beta gene expression in cultured fibroblasts and keratinocytes from burned patients. Acta Cir Bras 28: 551-558, 2013.

26. Serra MB, Barroso WA, da Silva NN, Silva SDN, Borges ACR, Abreu IC and Borges MODR: From inflammation to current and alternative therapies involved in wound healing. Int J Inflam 2017: 3406215, 2017.

27. Mahlapuu M, Hakansson J, Ringstad L and Björn C: Antimicrobial peptides: An emerging category of therapeutic agents. Front Cell Infect Microbiol 6: 194, 2016.

28. Sung YY, Kim YS and Kim HK: Illicium verum extract inhibit TNF- $\alpha$ - and IFN- $\gamma$-induced expression of chemokines and cytokines in human keratinocytes. J Ethnopharmacol 144: 182-189, 2012.

29. Yano S, Komine M, Fujimoto M, Okochi H and Tamaki K: Interleukin 15 induces the signals of epidermal proliferation through ERK and PI 3-kinase in a human epidermal keratinocyte cell line, HaCaT. Biochem Biophys Res Commun 301: 841-847, 2003.

This work is licensed under a Creative Commons Attribution-NonCommercial-NoDerivatives 4.0 International (CC BY-NC-ND 4.0) License. 